\title{
DEMOCRACIA CUÁNTICA: APUNTES SOBRE LA RECONSIDERACIÓN DEL TERRITORIO EN LA POLÍTICA DEMOCRÁTICA CONTEMPORÁNEA
}

\author{
QUANTUM DEMOCRACY: NOTES ON RETHINKING TERRITORY IN \\ CONTEMPORARY DEMOCRATIC POLITICS
}

\author{
John Keane ${ }^{1}$ \\ Universidad de Sidney (Australia)
}

Recibido: 30-11-2014

Aceptado: 02-02-2015

Resumen: Las crisis políticas suelen describirse como súbitos puntos de inflexión, momentos de dramatismo extremo, puntos críticos en los que todo está en juego, en los que se necesitan juicios y decisiones audaces. Sin embargo, sabemos por arqueólogos, paleontólogos, historiadores y otros expertos que la transformación y/o el derrumbe de los órdenes antiguos y su sustitución por nuevas organizaciones de poder suelen producirse con lentitud. Su ritmo es el de la larga duración, lo que significa que sus consecuencias radicales requieren tiempo para materializarse. Las rupturas que se producen lentamente son mucho más difíciles de detectar, y aún más de analizar, pero deben ser una parte central del análisis de la democracia contemporánea y de su futuro incierto, o al menos eso es lo que argumenta el proyecto de la democracia cuántica.

Palabras-clave: democracia, crisis, democracia cuántica, política, territorio.

\begin{abstract}
Political crises are normally described as sudden turning points, moments of gripping drama, flashpoints when everything is up for grabs, when bold judgments and decisions become necessary. But we know from archaeologists, paleontologists, historians and others that the radical transformation and/or ruination of old orders and their replacement by new power arrangements often happen slowly. Their rhythm is that of the longue durée, and that means their radical consequences take time to materialise. Slow-motion ruptures are much harder to spot, let alone to analyse, but they must be central to the analysis of contemporary democracy and its uncertain future, or so the Quantum Democracy project argues.
\end{abstract}

Key-words: democracy, crisis, quantum democracy, politics, territory.

[1] (john.keane@sydney.edu.au) Profesor de filosofía política en la Universidad de Sidney y del Centro Científico para la Investigación Social de Berlín. En España es conocido especialmente por sus libros Democracia y Sociedad Civil (1992) y The Life and Death of Democracy (2009). 


\section{Introducción ${ }^{2}$}

La hipótesis de arranque de la democracia cuántica es que los ideales y las instituciones democráticas están atrapados en una profunda crisis cuyas raíces pueden encontrarse en el mero hecho de que las democracias de los Estados nación son actualmente postsoberanas y se encuentran atrapadas por una miscelánea de instituciones ostentadoras de poder diseñadas para producir y administrar decisiones que no están vinculadas en un sentido simple al territorio. La política de las actuales democracias, enredada en redes globales de interdependencias respaldadas por flujos de comunicación mediática que reducen las distancias y el tiempo, ya no se encuentra en espléndido aislamiento del resto del mundo. Las controversias respecto a los límites, los efectos indirectos, las presiones del arbitraje y los efectos mariposa son frecuentes. La dialéctica entre el aquí y el allí es algo crónico. Los acontecimientos políticos de un lugar tienen efectos en otras partes del mundo, en lugares lejanos. La dinámica inversa es también habitual: los acontecimientos, flujos de información, declaraciones y acuerdos que suceden en lugares lejanos pueden tener, y de hecho tienen, consecuencias locales inmediatas.

Si esto es así, una pregunta clave es si la democracia puede ser reconcebida de manera caleidoscópica, es decir, entendida en términos cuánticos de múltiples marcos espaciales en interacción en los que los pueblos, con la ayuda de sus representantes, se gobiernan a sí mismos y sus ecosistemas en entornos de tamaños diversos marcados por ritmos espacio-temporales diferentes. El proyecto de la democracia cuántica supone que, en la actualidad, la teoría democrática necesita con urgencia un salto cuántico, un importante avance, un equivalente teórico al repentino paso de una partícula de un nivel de energía a otro. Cuestiona radicalmente el imaginario territorial dominante, basado en los lugares, de la política democrática contemporánea. Centra su atención en explicar por qué las cuestiones de territorio, lugar y espacio importan en la democracia, por qué tienen que trasladarse al centro de la teoría democrática, y por qué el interés cuántico en cuestiones como las "dimensiones extra», el entrelazamiento cuántico y el «espacio-tiempo» son tan relevantes para la política democrática. El proyecto explora las debilidades conceptuales y empíricas de las perspectivas de la "democracia cosmopolita», las teorías de la "demoicracia» y otras tentativas actuales de encontrar un lenguaje posterritorial para la democracia. Explora por qué, contra lo que podría esperarse, el espíritu, el lenguaje y las instituciones de la democracia sobreviven y prosperan hoy en día en contextos transfronterizos, y por qué el futuro de la democracia del siglo xxi depende de forma crucial de que se desafíe el estancamiento académico mediante el desarrollo de una poética cuántica y una política práctica del espacio democrático.

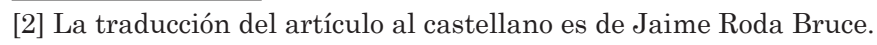

THÉMATA. Revista de Filosofía, Nº52 julio-diciembre (2015) pp.: 15-30 doi: 10.12795/themata.2015.i52.01 


\section{Contexto}

La hipótesis de arranque del proyecto de democracia cuántica que se esboza a continuación es que los ideales y las instituciones democráticas están atrapados en una profunda crisis cuyas raíces pueden encontrarse en el mero hecho de que las democracias de los Estados nación son actualmente postsoberanas y se encuentran atrapadas por una miscelánea de instituciones ostentadoras de poder diseñadas para producir y administrar decisiones que no están vinculadas en un sentido simple al territorio. La política de las actuales democracias, enredada en redes globales de interdependencias respaldadas por flujos de comunicación mediática que reducen las distancias y el tiempo (Keane, 2013; Khanna, 2014), ya no se encuentra en espléndido aislamiento del resto del mundo. La ausencia de linealidad es la nueva norma. Las controversias respecto a los límites, los efectos indirectos, las presiones de arbitraje y los efectos mariposa son frecuentes. La dialéctica entre el aquí y el allí es algo crónico. Los acontecimientos políticos de un lugar tienen efectos en otras partes del mundo, en lugares lejanos. La dinámica inversa es habitual: los acontecimientos, flujos de información, declaraciones y acuerdos que suceden en lugares lejanos pueden tener, y de hecho tienen, consecuencias locales inmediatas.

El proyecto de la democracia cuántica examinará y catalogará las múltiples formas en las que los mecanismos democráticos del «interior» son modelados y transformados cada vez más por fuerzas del «exterior». No es solo que las democracias de los Estados nación se enfrenten a fardos de problemas cada vez más pesados que únicamente pueden resolverse a través de la ayuda externa, o que las democracias estén ahora tan interrelacionadas entre sí que habitualmente comenten sobre sus asuntos internos, copien sus políticas e instituciones, negocien sus desacuerdos y aprendan de los errores mutuos. También están sucediendo otras cosas menos positivas. La intensificación de la integración mundial de los Estados y los pueblos significa que, en cualquier democracia, aquello que los ciudadanos y sus representantes de gobierno deciden en el «interior» de sus Estados conforma y, a menudo, perjudica a los pueblos del "exterior», que tienen limitadas posibilidades de reparación o indemnización. En estas circunstancias, el viejo axioma de que «toda democracia real se basa en el principio de que no solo los iguales son iguales sino que los desiguales no son tratados igualitariamente» (Schmitt, 1988, 9) asume un nuevo y variable significado, puesto que cuando la democracia, en su forma alemana o estadounidense por ejemplo, produce injusticias en otros pueblos distantes, haciéndoles sufrir decisiones, o indecisiones, que los perjudican y sobre las que no tienen ningún control, esta democracia se convierte en un ejercicio de hipocresía en el que se dispensa el poder de manera arbitraria. Por último, en tanto que las democracias se encuentren atrapadas en campos de fuerza transfronterizos, las opciones políticas se encontrarán restringidas y las decisiones

THÉMATA. Revista de Filosofía, ${ }^{\circ} 52$ julio-diciembre (2015) pp.: 15-30 doi: 10.12795/themata.2015.i52.01 
políticas se frustrarán o bloquearán por completo. Asuntos tales como la inmigración, la banca, los tipos de cambio, el turismo, la educación, y las fuerzas policiales y militares son ahora regularmente codecididas por otros a distancia, con independencia de lo que las legislaturas, los votantes y los líderes de cada país deseen o decidan. La consecuencia es que parece haber cada vez menos «soberanía» en las democracias territoriales. En circunstancias extremas, las disfunciones de la expansión multilateral de los acuerdos transfronterizos representan amenazas para las democracias, como cuando los bancos malos, el aire viciado, la violencia o las fugas nucleares de un país perjudican las democracias de sus vecinos. Incluso hay casos de pequeños Estados insulares (como las democracias de Kiribati y Tuvalu), donde el daño medioambiental amenaza con desplazar a los ciudadanos a nuevos territorios, desde donde intentarían ejercer un control jurisdiccional a distancia sobre sus lugares de origen.

En el ámbito la teoría democrática, los investigadores convencidos de que la democracia tiene lugar únicamente en contextos fijos delimitados territorialmente suelen ignorar o tratar como excepciones aleatorias estas anomalías empíricas y complicaciones de la vida real (Przeworski, 2010; Munck, 2011). Para algunos académicos, el espacio territorial no solo sigue siendo central en las cuestiones de poder y política, sino que le confieren una importancia creciente (Therborn, 2006). Los estudiosos de la democracia, reforzados por metodologías que se dan por sentadas y por inversiones considerables en bases de datos «nacionales» estilo Freedom House, piensan inevitablemente en términos de contextos vinculados con lugares fijos, tales como las comunidades rurales, los barrios urbanos y los Estados territoriales. Su mentalidad territorial es muy evidente en las descripciones académicas contemporáneas de los requisitos funcionales de la democracia, que supuestamente incluyen: a) un estado territorial "soberano", definido territorialmente, que garantice a través de sus diversas subunidades la seguridad física de una población residente de ciudadanos sometidos al Estado de derecho; b) una cultura política compatible con los mecanismos electorales, que incluya la competencia entre los partidos políticos, unas elecciones periódicas y un gobierno parlamentario; c) una infraestructura social más o menos homogénea o «identidad nacional», consolidada por una lengua común, costumbres históricas comunes y un sentido común de territorio compartido; y d) una economía de mercado delimitada territorialmente capaz de generar suficiente riqueza como para sacar a los ciudadanos de la pobreza y garantizarles un nivel de vida básico suficiente para que puedan interesarse en los asuntos públicos y votar en elecciones periódicas.

El proyecto de la democracia cuántica explora las raíces primarias originadas en el siglo XVIII de esta comprensión de la democracia basada en un Estado territorialmente definido. Con ello pretende poner de relieve la creciente extrañeza del concepto en el siglo xxi. La hipótesis es que la democracia representativa "posibilitada [...] por una gran extensión de territorio» (Des- 
tutt de Tracy, 1811, 19) fue un logro singularmente moderno. Desde cualquier perspectiva de análisis, se trataba de una forma extraordinariamente novedosa de autogobierno, un sistema gubernamental definido por constituciones escritas, poderes judiciales independientes y leyes que garantizaban procedimientos tales como la elección periódica de los candidatos de las asambleas legislativas, la duración limitada de los cargos políticos, la votación secreta, la competencia de los partidos políticos, el derecho a reunirse públicamente y la libertad de prensa. En este sentido, la democracia representativa amplió considerablemente la escala geográfica de la vida política. A medida que pasaba el tiempo, a pesar de su localización original en ciudades, distritos rurales y contextos imperiales de gran escala, la democracia fue circunscribiéndose a Estados definidos territorialmente respaldados por poderes administrativos, legislativos y fiscales, así como por fuerzas policiales y ejércitos permanentes (Mann, 1993). Estos estados eran cualitativamente mayores y estaban más poblados que las unidades políticas de la democracia asamblearia. Las democracias del mundo clásico griego eran sistemas de pequeña escala. La mayoría de ellas, como, por ejemplo, Mantinea y Argos, no abarcaban más que algunas decenas de kilómetros cuadrados. De ahí la observación generalizada de que el salto de «la visión de la democracia en la ciudad-Estado que había prevalecido [...] desde los griegos hasta Rousseau» a la "solución académica autorizada [...], el Estado-nación» fue una verdadera revolución en el paradigma. Según la conclusión estándar: «El gobierno representativo del Estado-nación es, en muchos aspectos, tan radicalmente - e inevitablemente- diferente de la democracia en la ciudad-Estado que resulta casi una desventaja intelectual aplicar el mismo término, democracia, a ambos sistemas, o creer que en esencia son realmente lo mismo» (Dahl, 1967, 956).

\section{Cuestionamiento}

El proyecto de la democracia cuántica cuestiona críticamente esta visión ortodoxa de tres maneras. En primer lugar, plantea preguntas sobre las conexiones ocultas entre los modelos de la antigua democracia asamblearia y la «democracia territorial» moderna (Brownson, 1972). Es indiscutible que estos modelos se situaban en los extremos opuestos de un espectro de tamaño: los primeros eran versiones de pequeña escala y los segundos eran versiones de gran escala de la democracia. Sin embargo, el proyecto se pregunta si los dos modelos enfrentados de democracia no comparten la misma presunción de que el autogobierno popular está siempre, de manera "natural» e irrevocable, anclado en un lugar físico definido. ¿Podría ser que en las democracias, tanto en las antiguas como en las modernas, los espacios demarcados territorialmente sirvan como un marco fijo para los ciudadanos, para sus encuentros, deliberaciones y decisiones? Es posible que la democracia tenga cualidades telúricas, 
que el lugar sea un componente intrínseco. Puede que la democracia sea inseparable de su ubicación: el autogobierno mediante la pertenencia significativa a un lugar definido.

El proyecto está profundamente interesado en el hecho de que las perspectivas democráticas antiguas y modernas se encuentren atrapadas en una mentalidad basada en el lugar fijo. Explora los contornos de esta mentalidad y por qué las dificultades que tienen los demócratas en pensar más allá del lugar merecen reflexión crítica. El proyecto investiga cómo las anteriores democracias «caseras» estaban típicamente ligadas al territorio (de terra: tierra, terreno, alimentación, sustento). Se pregunta por qué se han utilizado a menudo como las guardianas del territorium (el lugar desde el que se advierte a las personas) y como el trampolín para la guerra y el terror (terrere). Examina en qué medida opera aún la actual democracia en lugares físicos demarcados, sea en las asambleas de las capitales o las asambleas locales (la versión moderna de la pnyx de Atenas), los Stadtstaaten (Berlín y Hamburgo), los territorios de la capital, los distritos y provincias de los Estados federales, o el territorio del Estado en su totalidad. Estos lugares son, según la hipótesis del proyecto, los lugares privilegiados elegidos. En algunos casos, como en las cámaras superiores de los sistemas bicamerales, gozan de derechos de representación. El lugar geográfico es «donde» sucede la democracia, por lo que se supone una visión «localizadora». El topos y el anclaje de la pertenencia significativa y el apego afectivo pueden definirse mediante criterios como la distancia mensurable, la latitud y la longitud, o la elevación. El lugar es una sección tridimensional de la tierra o el mar que no es equivalente a ninguna otra. El lugar está acotado, es una unidad «natural», un lugar fijo donde "suceden» las cosas democráticas, donde el poder se ejerce, sujeto al consentimiento de los ciudadanos con derecho a voto, por medio de sus representantes y a través de las instituciones. El lugar es la dirección de la democracia, la dirección donde habita la democracia.

En segundo lugar, el proyecto plantea preguntas que señalan una contradicción fundamental: ¿si la democracia significa mínimamente una forma política que permite a las personas gobernarse a sí mismas, tomar control sobre sus vidas, en un espacio de tierra determinado, no está entonces la democracia perdiendo terreno rápidamente ante las múltiples dinámicas de las cadenas transfronterizas de poder empresarial, legal y gubernamental que no rinden cuentas públicamente? ¿Estas dinámicas transfronterizas no arremeten profundamente contra la esencia, tanto en términos de significado (semántica) como de eficacia (pragmática), del concepto de democracia basada en el lugar? ¿Podrían señalar su obsolescencia como gran ideal político? Estas preguntas se utilizarán para introducir en el análisis diversas abreacciones, que van desde el silencio sombrío y las reacciones públicas violentas a los esfuerzos académicos por reexaminar el significado de la democracia en los contextos transfronterizos. Se investigarán cinco tipos de respuesta a dicha contradicción: 
El fatalismo. Supone que poco o nada puede hacerse para deshacer o suavizar la contradicción. Las iniciativas ciudadanas, las innovaciones democráticas y las discusiones sobre la democracia transfronteriza son invalidadas por las dinámicas transfronterizas. La democracia es una florecilla indefensa, una víctima del avance del «espacio abstracto» (Lefebvre, 1991) impuesto por los organismos públicos y empresariales transfronterizos. Se dice que las perspectivas de la democracia se han debilitado todavía más por la hipercomplejidad de los métodos de toma de decisiones transfronterizos, que imposibilita la «ilustración del público» y «el discurso y compromiso civiles» tan necesarios para la democracia (Dahl, 1999).

La reconstrucción de la democracia territorial. Se supone que los políticos, los partidos políticos, los votantes y los gobiernos pueden combinar sus fuerzas para volver atrás en el tiempo y apuntalar los muros de la fortaleza de la democracia parlamentaria «recuperando» los poderes transfronterizos para el Estado territorial. Las instituciones del Estado, reforzadas, deben convertirse de nuevo en las principales guardianas del principio democrático de la territorialidad soberana respaldada por «el pueblo».

La recuperación de lo local. Se busca revivir el autogobierno local de las personas y, de hecho, se quiere hacer recuperando el espíritu (imaginado) de la democracia asamblearia de estilo griego. Algunos estudiosos prevén una nueva geografía de «ciudades abiertas» y «ciudades refugio» (Derrida, 2001). Otros reviven el discurso del "poder para el pueblo», el lema utilizado por grupos como los Students for a Democratic Society (Estudiantes por una Sociedad Democrática) durante las rebeliones de 1960, con el fin de demostrar que el autogobierno requiere una toma de decisiones descentralizada y la autodeterminación de los ciudadanos a través de formas innovadoras de democracia "profunda" de «base popular» que sirvan para aumentar «el compromiso y la capacidad de la gente corriente para tomar decisiones sensatas a través de una deliberación razonada y empoderada, puesto que se pretende vincular la acción al debate» (Fung y Wright, 2003, 5; Barber, 2013).

El federalismo global. Se trata de una forma neokantiana de imaginar un "sistema de geogobierno distinto de cualquier otro que se haya propuesto hasta la fecha", un sistema de "democracia cosmopolita» de dos niveles en el que todos los pueblos gocen de determinados derechos humanos básicos y sean tratados como fines en sí mismos, gracias a «un modelo de organización política en la que los ciudadanos, sin importar su ubicación en el mundo, tengan una voz, una aportación y una representación política en los asuntos internacionales, en paralelo a la de sus propios gobiernos y con independencia de ellos» (Archibugi y Held, 1-16; Habermas, 2008).

La demoicracia. Concibe un conjunto de estados gobernados democráticamente (van Parijs, 1998; Nicolaïdis, 2012; Cheneval y Schimmelfennig, 2013). Esta perspectiva, que se utiliza principalmente para describir y legi-

THÉMATA. Revista de Filosofía, No52 julio-diciembre (2015) pp.: 15-30 doi: 10.12795/themata.2015.i52.01 
timar la Unión Europea, recomienda una forma de gobierno compuesta por sistemas gubernamentales responsables ante múltiples pueblos distintos (demoi). Esta perspectiva supone que el gobierno transfronterizo en su forma democrática no necesita cimentarse sobre la identidad común de un único pueblo «soberano». La demoicracia es un nuevo tipo de "confederación», una forma de gobierno que valora el pluralismo, insta al reconocimiento público mutuo de las diferencias, y reconoce tanto la creciente interdependencia de los Estados y los pueblos como la posibilidad de que varios pueblos separados puedan trabajar conjuntamente para limitar y legitimar de manera efectiva el poder de las instituciones de gobierno transfronterizas.

En tercer lugar, el proyecto de la democracia cuántica estudia detenidamente estos diferentes enfoques preguntándose si, paradójicamente, su efecto combinado puede ser el de deshabilitar la imaginación democrática, esencialmente porque todos ellos están vinculados a una u otra versión de la comprensión territorial, basada en el lugar, de la democracia. Todas consideran la democracia como una forma de autogobierno popular, pero el proyecto se pregunta si no estarán todas ellas tácitamente comprometidas con una visión homogénea de la democracia como algo asentado en un lugar físico fijo. Podrían ser esfuerzos de resistencia y reparación sustancial pegados como parches sobre el decrépito edificio de la democracia territorial. Si este es el caso, sus desacuerdos podrían verse como algo interno, en el sentido de que tendrían que ver con cuestiones de viabilidad y tamaño: por ejemplo, ¿cuál es el tamaño geográfico óptimo para una democracia? ¿Cuáles son las ventajas y desventajas comparativas de los sistemas democráticos según su magnitud? ¿Puede un sistema de democracia representativa ser demasiado grande para que puedan controlarlo sus ciudadanos? (Dahl y Tufte, 1973).

\section{Un giro cuántico}

La principal dificultad que plantean estas preguntas de sentido común, de "caja china», respecto al lugar y el tamaño, al menos según la hipótesis central del proyecto, es su incapacidad para aprehender las múltiples dinámicas espacio-temporales que hoy en día conforman, debilitan y revitalizan simultáneamente todas las democracias del mundo real. En este punto, el proyecto pretende dar un giro cuántico, embarcándose inicialmente en el aprendizaje de las perspectivas, los conceptos y los métodos desarrollados por los apasionantes ámbitos de investigación de la cosmología contemporánea y la física de partículas. Sus perspectivas cuánticas se utilizarán como catalizadores para plantear preguntas sobre las muchas formas en que la mentalidad de la democracia territorial resulta insuficientemente compleja y (obstinadamente) ignorante de la "rareza cuántica» (Albert, 1992) de unos fenómenos que requieren un replanteamiento fundamental de la democracia tal y como la hemos conocido. 
En cuestiones de democracia, el proyecto de la democracia cuántica pretende mostrar cómo están sucediendo cosas que no solo son más extrañas de lo que se pensaba en el pasado, sino que son mucho más extrañas de lo que podríamos pensar en la actualidad. Atrapada en un estancamiento académico, la democracia territorial, con su sentido común y su fijación tridimensional en la latitud, la longitud y la altitud, ignora el multiverso en expansión de unidades democráticas de distintos tamaños entrelazadas espacialmente. Pasa por alto su dinamismo multidimensional, sus complejos patrones de interacción en campos de poder interconectados que se extienden en todas las direcciones, de lo local a lo global y más allá, y viceversa. Para expresarlo metafóricamente en lenguaje cuántico, el proyecto se pregunta si las teorías de la democracia territorial no estarán ignorando fenómenos que incluirían desde los quarks, los neutrinos y otras partículas elementales hasta los planetas, las estrellas, las zonas de energía oscura, las enanas rojas y exoplanetas del tamaño de la Tierra que se encuentran en la galaxia en rápida expansión (y en proceso de desaparición) que conocemos. El proyecto va aún más lejos: sugiere que los modelos de democracia territorial no logran comprender que estos cuantos de alta energía operan en más de tres dimensiones y están en constante movimiento, vibración y oscilación en los campos cuánticos, empujando y tirando en múltiples direcciones, a menudo desafiando la gravedad, ampliando el universo de la democracia e impulsándolo hacia destinos desconocidos (Weinberg, 1995).

El proyecto está profundamente interesado en las complejas incertidumbres que generan estos procesos cuánticos. Aunque tales indeterminaciones parecen imposibilitar las formulaciones y los cálculos exactos, las teorías y observaciones son necesarias y factibles, como lo son en los campos de investigación de la cosmología contemporánea y la física de partículas. La teoría democrática contemporánea se enfrenta a una cuota de "materia oscura» no descubierta mayor de la que le corresponde, sin embargo, desde el punto de vista de este proyecto, las desconcertantes incógnitas no deben impedirle cartografiar los contornos de la democracia actual con parámetros cuánticos. De un modo extraño, estas incógnitas albergan soluciones: generan perplejidad, pero a la vez motivan nuevas preguntas, nuevas formas de pensar y nuevas soluciones a raras anomalías y problemas sin resolver.

\section{Estudios de casos}

Paralelamente al inventario y disección de la mentalidad territorial, el proyecto de la democracia cuántica examina varios nuevos tipos de instituciones democráticas actuales que resultan anómalos cuando se miden con los viejos parámetros de la democracia territorial. El proyecto sostiene que los tres tipos básicos elegidos para la investigación son indicativos de una tendencia 
mucho más amplia. En términos históricos, estos fenómenos "cuánticos» no tienen precedentes: pertenecen de lleno a la era de la democracia monitorizada (Keane, 2009) y su importancia teórica y práctica, según espera demostrar el proyecto, radica en su abierto desafío a la mentalidad territorial que continúa dominando los escritos sobre democracia.

La biodemocracia. Se refiere a formas de autogobierno que consideran a las personas, los animales, los árboles, las rocas y los ríos como elementos entrelazados en un tejido común y, por lo tanto, rechazan la presunción de que la tierra en la que viven las personas es simplemente un "territorio» definible, entendido como un recurso que los ciudadanos y sus representantes pueden explotar. Igual que la investigación de la física de partículas se enfrentó con éxito a la presunción de que el átomo era el elemento último de construcción del universo, los experimentos en biodemocracia se consideran algo integrado en redes dinámicas vivas de interacciones humanas que penetran «profundamente» en el mundo de lo no humano, y viceversa. El proyecto prevé realizar una profunda investigación de campo sobre varios casos, entre los que se incluyen los experimentos creadores de tendencia del autogobierno indígena en el archipiélago Haida Gwaii (Islas del Pueblo) del nordeste del Pacífico y el parque nacional Uluru-Kata Tjuta del centro de Australia. El trabajo de campo comparará, contrastará y evaluará la importancia política general de cómo estos experimentos democráticos se encuentran profundamente impregnados de las nociones de a) la complejidad frágil y dinámica del mundo; b) la interconectividad y el potencial de autoorganización de todos los elementos vivos y no vivos del entorno político; c) el profundo respeto por lo no humano y su «derecho de representación» legítimo en los asuntos humanos; d) el conocimiento de los efectos no lineales y, en consecuencia, de la indeterminación crónica y la potencial inestabilidad de los esfuerzos humanos de autogobierno en los entornos ecológicos; e) la importancia fundamental de los consejos, los foros de patrimonio cultural y otros métodos de decisión política para rechazar el poder arbitrario y promover el conocimiento público de todas estas cualidades, así como para potenciar las capacidades de gestión eficaz de los resultados no lineales y potencialmente inestables; y f) la dificultad de establecer límites estrictos entre los pueblos «locales» y «distantes» y las instituciones políticas, de lo que se deriva el imperativo cuántico de pensar en la democracia en términos de espacios múltiples cuyos horizontes se extienden mucho más allá del contexto de la «patria».

El autogobierno transfronterizo. Algunos nuevos mecanismos democráticos operan en contextos transfronterizos usando ingeniosos métodos para vincular entre sí lugares geográficos remotos en una gran diversidad de husos horarios. El proyecto de la democracia cuántica llevará a cabo un minucioso análisis del Forest Stewardship Council (FSC) con sede en Bonn, una organización internacional de la sociedad civil constituida por múltiples partes intere- 
sadas fundada a principios de 1990 para promover la gestión responsable de los bosques del mundo (Humphreys, 1996). El FSC, compuesto por una red mundial de socios descentralizada, es un organismo autónomo de adhesión abierta comprometido con la «participación, la democracia, la equidad y la transparencia». Tiene tres cámaras (social, económica y medioambiental) y cada uno de los miembros pertenece a una de ellas. Sus participantes, tanto del hemisferio del norte como del hemisferio sur, gozan de iguales derechos de representación y voto en el órgano supremo de adopción de decisiones, la Asamblea General. El proyecto sugiere que el FSC podría ser un buen ejemplo de método a distancia para el manejo transfronterizo de la responsabilidad de poder, un tipo de organización política que decide quién recibe qué, así como cuándo y cómo lo recibe, en unos espacios multidimensionales no vinculados al territorio en un sentido directo y tridimensional. El FSC, comprometido con el autogobierno y consciente de los peligros del poder arbitrario, hace algo más que abrazar una perspectiva cuántica de «mundos múltiples»: supone una interrelación dinámica entre estos espacios. Asume que el movimiento es algo inherente en estos mundos múltiples interconectados; lo que sucede en un espacio se encuentra constantemente modelado por las cosas que suceden en otros espacios. El entrelazamiento cuántico es algo crónico.

$\mathrm{Al}$ analizar las nuevas formas de autogobierno transfronterizo, el proyecto de la democracia cuántica se pregunta si viejos dualismos como tiempo/lugar y tiempo/espacio, no han dejado simplemente de tener sentido. ¿Es posible que no sea el territorio, sino múltiples campos de espacio-tiempo lo que ahora conforme el marco de orientación cuántica de estos nuevos experimentos democráticos, algunos de los cuales carecen de sede fija o dirección permanente? En la búsqueda de una respuesta a esta pregunta, el sistema de gobierno conocido como la Administración Central Tibetana (ACT) tiene una gran pertinencia para el proyecto. Algunos estudiosos lo han comparado despectivamente con un aeropuerto o una autopista, un "no lugar» (Augé, 1995), sin embargo, en realidad, su arquitectura en el espacio-tiempo rezuma rareza cuántica. El trabajo de campo propuesto tiene que lidiar con una nueva forma de autogobierno parlamentario que va más allá del territorio. Aunque la ACT opera actualmente en el norte de la India, su "patria» está en otra parte, cruza fronteras y se encuentra en otros lugares geográficos («el Tíbet histórico»). El sistema de gobierno opera en el espacio de otro tiempo: una patria imaginaria en un futuro lejano. El poder legislativo reside en el parlamento de la ACT, que es elegido por votación popular cada cinco años por ciudadanos exiliados que viven en muchos países extranjeros distintos. Sus miembros están en constante movimiento. Las distintas circunscripciones geográficas (diputados de Europa y América del Norte) y agrupaciones funcionales (diferentes escuelas del budismo y la fe prebudista Bon) comparten los derechos de representación en la legislatura. De conformidad con

THÉMATA. Revista de Filosofía, No52 julio-diciembre (2015) pp.: 15-30 doi: 10.12795/themata.2015.i52.01 
una constitución escrita, se concede la ciudadanía a cualquier persona nacida en la patria futura imaginaria (o a sus descendientes). En la práctica, puesto que a menudo resulta difícil demostrar este lugar de nacimiento, la ciudadanía se establece mediante entrevistas, que se realizan en alguna de las muchas oficinas de gobierno que hay en todo el mundo. Muchos ciudadanos consideran sus vidas como algo inconstante, como algo permanentemente efímero, y, según la hipótesis de este proyecto, este es el motivo por el que valoran la democracia. La democracia, cuyo principal representante es el $14^{\circ}$ Dalai Lama, se ve como algo más que la máxima expresión de la igualdad de los individuos y la preocupación por todos los seres humanos y no humanos, se considera la guardiana de la impermanencia, una advertencia sobre los peligros del apego (al poder), la garante del flujo y la fluidez, un recuerdo vivo para los ciudadanos de que pertenecen a un mundo futuro prefigurado por un mundo presente en el que nada es esencialmente sí mismo.

Las elecciones móviles. El proyecto de la democracia cuántica tendrá que hacer frente a la posible objeción de que la biodemocracia y el autogobierno transfronterizo con características cuánticas son de importancia académica marginal, al menos en comparación con la presunta fuente de la democracia: las elecciones periódicas. Según el argumento ortodoxo, el eje de la democracia contemporánea siguen siendo las elecciones en contextos territoriales fijos. El proyecto explorará una hipótesis contrapuesta: que las elecciones están cada vez más marcadas por características cuánticas. Las campañas, los votantes y los partidos políticos, por ejemplo, sienten regularmente la punzada de lo que los físicos cuánticos (Bohm, 1980) llaman el principio de no localidad: del mismo modo que la manipulación experimental de una partícula altera instantáneamente el comportamiento de sus partículas hermanas en otros lugares, los efectos no locales se entretejen en el universo de las elecciones. El proyecto cuestiona la mentalidad territorial de la investigación académica existente sobre las «fluctuaciones internacionales de la opinión» y el «efecto de vecindario» de las elecciones (Kim y Fording, 2001). Tomará nota de la dinámica cuántica de las elecciones transfronterizas en los sistemas de gobierno multiestatales (un prototipo evidente son las elecciones al Parlamento Europeo), y de los procesos de votación en organizaciones internacionales de la sociedad civil, como el Comité Olímpico Internacional, cuyos organismos ejecutivos están sujetos a elección por votación secreta, por mayoría de votos emitidos, para mandatos de duración limitada. El proyecto está especialmente interesado en los efectos modeladores de los medios digitales y la abundancia comunicativa (Castells, 2012; Keane, 2013), sobre todo en las oportunidades que ofrecen a los responsables de las campañas, los periodistas y los supervisores de las elecciones para intervenir desde la distancia en las elecciones locales, convirtiéndolas de esta manera en asuntos no locales. Se prestará atención a la historia prácticamente indocumentada de los múltiples procesos mediante los cuales, desde 
1960, las elecciones presidenciales de los Estados Unidos se han visto profundamente envueltas (implicadas, como dirían los expertos en física cuántica) en los asuntos de otros sistemas de gobierno. Los casos de elecciones estatales territoriales cuyas dinámicas locales son moldeadas en tiempo real por campañas a distancia no locales son de gran interés para el proyecto de la democracia cuántica. Un ejemplo notable fueron las elecciones generales que se disputaron ferozmente en Malasia en 2013. El uso por primera vez del voto por correo para todos los malasios que se encontraban en el extranjero provocó lo que los físicos llaman el «efecto de túnel cuántico»: ciudadanos, candidatos, responsables de campañas, personal de la comisión electoral, flashmobs y supervisores de las elecciones de Malasia que se movían en todas las direcciones, viajando y enviando mensajes a través de las fronteras, comportándose de maneras no locales, en contextos lejanos desde Singapur y el sur de Tailandia hasta Kalimantan en Indonesia.

\section{Implicaciones para la investigación}

La conjetura que guía el proyecto es que las perspectivas cuánticas ofrecen ventajas descriptivas, estratégicas y normativas a una democracia cuyo futuro se encuentra en peligro. $\mathrm{Al}$ aprovechar las actitudes, metáforas y conceptos derivados de campos de estudio como la física de partículas y la cosmología, el proyecto de la democracia cuántica representa, como mínimo, un ejercicio de revitalización del imaginario democrático. En términos descriptivos, liberará a la política democrática de las garras de la psefocracia, la gastada presunción de que, a fin de cuentas, la democracia se limita en última instancia a unas elecciones libres y justas, a la votación periódica por parte de los ciudadanos de una nación en entornos definidos territorialmente. El proyecto implica una redefinición estratégica y normativa de la democracia como mucho más, aunque nada menos, que unas elecciones libres y justas. Tiene un carácter de urgencia. Realiza un inventario de las formas en que el espíritu, el lenguaje y las instituciones de la democracia en su forma de estado territorial están siendo perturbadas y «desbordadas" por una diversidad de causas y causantes transfronterizos. El proyecto describe las alternativas democráticas que están emergiendo. Propone que una perspectiva cuántica, con énfasis en espacios de diferentes tamaños dinámicamente interconectados, está mejor equipada para ofrecer a la democracia una buena oportunidad de supervivencia en un mundo en el que los tratados jurídicos organizados por los Estados se encuentran cada vez más entrelazados con entidades universales como las Naciones Unidas, las redes de organizaciones regionales, los acuerdos «minilaterales», los códigos de conducta transfronterizos, las asociaciones con actores no gubernamentales, los acuerdos informales y los experimentos de políticas fragmentarios. El proyecto de la democracia cuántica podría tener efectos perturbadores. Cuestiona las presunciones científicas con-

THÉMATA. Revista de Filosofía, No52 julio-diciembre (2015) pp.: 15-30 doi: 10.12795/themata.2015.i52.01 
vencionales de que las teorías de la democracia transfronteriza están destinadas a fracasar porque chocan contra problemas tan inabordables como la ignorancia del público sobre los asuntos globales y la imposibilidad de reunir a los ciudadanos como partes interesadas en igualdad de condiciones, sin la tiranía de la mayoría, a través de las fronteras territoriales (Christiano, 2006). El proyecto trata de mostrar, por el contrario, por qué el confinamiento de la democracia en los barracones del Estado produce estos efectos discapacitantes en la imaginación democrática, y cómo esto puede remediarse positivamente. El enfoque cuántico plantea una pregunta importante y sustancial: si en las primeras décadas del siglo XxI es posible redefinir la democracia, para llevar a cabo su «desterritorialización», dejarla libre en el mundo y pensar en ella como un proyecto político infinito a través del cual las personas humanas y sus representantes buscan conjuntamente el control público del ejercicio del poder, donde quiera que se ejerza, en un multiverso de ubicaciones espacio-temporales interconectadas.

La elección de los estudios de caso y el énfasis en la posibilidad de construir una teoría de la democracia con características cuánticas en una era de postsoberanía ofrecen nuevas formas de asegurar que el espíritu y la esencia de la democracia puedan sobrevivir y prosperar bajo la presión de su creciente entrelazamiento con redes de poder arbitrario respaldadas por flujos de comunicación mediática que reducen las distancias y el tiempo. La teoría cuántica de la democracia no debe confundirse con las soluciones de "constitución mixta» basadas en el lugar (Polibio). La teoría cuántica es más dinámica, más radicalmente plural, tiene más recursos y está más alerta a las contradicciones y las autocontradicciones. En la región de Asia y el Pacífico, por ejemplo, y en el orden mundial en desarrollo, el concepto global de la democracia cuántica se encuentra bastante a gusto en los ámbitos entrelazados de la toma de decisiones, los conflictos de demarcación de fronteras y las luchas de poder. Su preocupación por la dinámica espacio-temporal la hace extremadamente sensible a la preocupante ausencia de mecanismos democráticos en ámbitos políticos clave como el sector bancario y de crédito, la industria armamentística mundial, los nuevos acuerdos comerciales estilo TPP y las solicitudes de asilo. En estos ámbitos de poder, centra su atención en una mayor responsabilidad pública de los que gobiernan sobre los otros o sobre los biomas en los que viven. El enfoque cuántico reconoce que un número creciente de problemas políticos pueden resolverse de manera abierta y justa solo en y entre los espacios subestatales, regionales y mundiales que están en perpetuo movimiento. Aboga, por tanto, por una comprensión "policéntrica» de la democracia. Supone que una fructífera combinación de esfuerzos experimentales en lugares múltiples, unidos entre sí mediante flujos de información libre y una intensa supervisión pública del poder, tienen, en su conjunto, claras ventajas para la resolución de problemas frente a otros métodos políticos más centralizados (Ostrom, 2009). 
Democracia cuántica: apuntes sobre la reconsideración del territorio en la política democrática contemporánea

\title{
Referencias bibliográficas:
}

\author{
Albert, David Z. (1992), Quantum Mechanics and Experience (Cambri- \\ dge, Mass.).
}

Archibugi, Daniele y Held, David (1995), Cosmopolitan Democracy: An Agenda for a New World Order (Cambridge).

Barber, Benjamin (2013), If Mayors Ruled the World: Dysfunctional Nations, Rising Cities (New Haven).

Bohm, David (1981), Wholeness and the Implicate Order (Londres).

Bohr, Niels (1958), Atomic Physics and Human Knowledge (Londres y Nueva York).

Castells, Manuel (2012), Networks of Outrage and Hope: Social Movements in the Internet Age (Cambridge).

Cheneval, Francis y Schimmelfennig, Frank (2013), «The Case for Demoicracy in the European Union», Journal of Common Market Studies, vol. 51, 2 (marzo) pp. $334-350$.

Christiano, Thomas, "A Democratic Theory of Territory and Some Puzzles about Global Democracy», Journal of Social Philosophy, vol. 37, 1 (primavera de 2006), pp. $81-107$.

Dahl, Robert A. (1967), "The City in the Future of Democracy», The American Political Science Review, vol. 61, 4, pp. 953 - 970.

Dahl, Robert A. y Tufte, E.R. (1974), Size and Democracy (Londres).

Derrida, Jacques (2001), On Cosmopolitanism and Forgiveness (Londres y Nueva York).

Destutt de Tracy (1811), A Commentary and Review of Montesquieu's Spirit of Laws (Filadelfia). York).

Dryzek, John (1990), Discursive Democracy (Cambridge y Nueva (2006), Deliberative Global Politics (Cambridge).

Eckersley, Robyn (2004), The Green State: Rethinking Democracy and Sovereignty (Cambridge, Mass.).

Fung, Archon y Wright, Erik Ohlin (2003), «Thinking about Empowered Participatory Governance», en Deepening Democracy: Institutional Innovations in Empowered Participatory Governance (Londres y Nueva York).

Goodin, Robert (2003), Reflective Democracy (Oxford y Nueva York). (2008), Innovating Democracy: Democratic Theory and Practice After the Deliberative Turn (Oxford y Nueva York).

Habermas, Jürgen (2008), «The Constitutionalization of International Law and the Legitimation Problems of a Constitution for World Society", Constellations, vol. 15, 4 (diciembre), pp. $444-455$. 
Heisenberg, Werner (1958), Physics and Philosophy: The Revolution in Modern Science (Londres).

Humphreys, David (2008), Forest Politics: the Evolution of International Cooperation (Londres).

Su Santidad el Dalai Lama, (1999), «Buddhism, Asian Values, and Democracy», Journal of Democracy, 10, 1, pp. 3-7. va York).

Keane, John (2009), The Life and Death of Democracy (Londres y NueNueva York). (2013), Democracy and Media Decadence (Cambridge y

Khanna, Parag (2014), Remapping the World (Londres y Nueva York, próxima publicación).

Kim, HeeMin y Fording, Richard C. (2001), «Voter Ideology, the Economy, and the International Environment in Western Democracies, 1952 1989», Political Behavior, 23, 1, pp. 53 - 73.

Lefebvre, Henri (1991), The Production of Space (Oxford).

Mann, Michael (1993), The Sources of Social Power: The Rise of Classes and Nation States, 1760 - 1914 (Cambridge y Nueva York).

Nicolaïdis, Kalypso (2012), "The Idea of European Demoicracy», en Julie Dickson y Pavlos Eleftheriadis (eds), Philosophical Foundations of European Union Law (Oxford), pp. 247 - 274.

Ostrom, Elinor (2009), A Polycentric Approach for Coping with Climate Change (Washington, DC).

Patapan, H y Kane, J (2012), The Democratic Leader: How Democracy Defines, Empowers and Limits its Leaders (Oxford).

Przeworski, Adam (2010), Democracy and the Limits of Self-Government (Nueva York).

Schmitt, Carl (1988), The Crisis of Parliamentary Democracy (Boston). (2003), The Nomos of the Earth in the International Law of the Jus Publicum Europaeum (Nueva York).

tung (Stuttgart).

(1954), Land und Meer: Eine Weltgeschichtliche Betrach-

Therborn, Göran (2006), «Why and How Place Matters», en Robert E. Goodin y Charles Tilly (eds.), Contextual Political Analysis (Oxford), pp. 509 -533 .

Weinberg, Steven (1995), The Quantum Theory of Fields, vol. 1 (Cambridge y Nueva York). 